Efficacy of exercise counselling as an aid for smoking cessation:

a randomized controlled trial

Michael Ussher*, Robert West*, Andy McEwen*, Adrian Taylor§, Andrew Steptoeđ.

*Department of Psychology, Hunter Wing, St. George's Hospital Medical School,

Cranmer Terrace, London SW17 ORE, UK.

§School of Physical Education, Sport and Leisure, DeMontfort University,

37 Landsdowne Road, Bedford MK40 3BZ, UK.

IDepartment of Epidemiology and Public Health, University College London,

2-16 Torrington Place, London WC1E 6BT, UK.

Address for correspondence and reprints:

Michael Ussher

Department of Psychology

Hunter Wing, St. George's Hospital Medical School,

University of London, Cranmer Terrace

London SW17 ORE, UK

Tel: (+44) 2087255605

Fax: (+44) 2087672741

Email: mussher@sghms.ac.uk <mailto:mussher@sghms.ac.uk>

Total word count: 5,236

Pages: 33 



\title{
Efficacy of exercise counselling as an aid for smoking cessation:
}

\section{a randomized controlled trial}

Michael Ussher, Robert West, Andy McEwen, Adrian Taylor, Andrew Steptoe

\begin{abstract}
Aim. To examine whether exercise counselling increases smoking abstinence and reduces tobacco withdrawal and gains in weight and body fat. Design. A randomised controlled trial. Setting. A community-based stop smoking clinic. Participants. 299 male and female smokers. Intervention. Participants were randomly assigned to a seven week smoking cessation programme including nicotine replacement therapy plus either (i) exercise counselling, or (ii) health education advice with equal contact time as for the exercise counselling condition. Measurements. Six weeks of smoking abstinence was confirmed by expired carbon monoxide,. Findings._There was no significant difference in smoking abstinence between the exercise group $(n=154)$ and the controls $(n=145)$ at six weeks $(39.6 \%$ versus $38.6 \%)$. Nor was there any difference in gains in weight or body fat, although those in the exercise group increased their exercise levels. Exercise participants reported less tension, anxiety and stress, and more happiness than the controls during the first week of smoking abstinence $(\mathrm{P}=.03$, $.01, .04, .03$, respectively); less irritability through two weeks of abstinence $(\mathrm{P}=.03)$, and less restlessness through three weeks of abstinence $(\mathrm{P}=.04)$. Conclusions. Adding brief exercise counselling to a smoking cessation programme did not significantly increase smoking abstinence or reduce gains in weight or body fat, although exercise levels were raised and there were some beneficial effects on psychological symptoms.
\end{abstract}

Key words: smoking cessation, intervention, exercise, physical activity 


\section{INTRODUCTION}

Cigarette smoking is a leading cause of premature death in industrialised countries (1) and smoking cessation extends life and reduces morbidity (2). A combination of behavioural support and nicotine replacement therapy (NRT) has been shown to be one of the most effective smoking cessation interventions, achieving abstinence rates at twelve months of approximately $15-20 \%$ (3). Additional interventions are needed in order to increase these success rates.

There is some evidence to suggest that exercise may be useful as an aid to smoking cessation (4). Of eight previous studies examining the efficacy of supervised, vigorous intensity exercise as an adjunct to a behaviourally-based smoking cessation programme three have found a significant benefit for those receiving an exercise programme (4). One of these studies examined the effect of thrice weekly sessions of vigorous (70-85\% of maximal heart rate) supervised aerobic exercise, lasting 30-45 minutes, on smoking abstinence in 20 female smokers (5). Following seven days of smoking abstinence significantly more of the exercise group remained abstinent when compared with a control group receiving only behavioural support. A second study examined the effect of four weeks of weekly vigorous (60-75\% of maximal heart rate) supervised exercise sessions lasting 15-45 minutes, plus three similar exercise sessions at home, on smoking rates in 205 male and female smokers recovering from an alcohol problem (6). At the end of treatment, but not after six or twelve months, rates of smoking abstinence were significantly higher for a group combining exercise and behavioural support than for those receiving nicotine gum and behavioural support, or having a standard smoking cessation treatment; although these results may not be generalizable beyond smokers with alcohol problems. 
The most methodologically rigorous study to date investigated the effect of 12 weeks of thrice weekly vigorous (60-85\% of heart rate reserve) supervised aerobic exercise, lasting 30-40 minutes, on smoking abstinence in 281 female smokers (7). Twelve months following treatment those in the exercise condition achieved approximately double the smoking abstinence rates of those in an equal contact control condition. In addition, those in the exercise condition gained significantly less weight at the end of treatment (7); and reported significantly less tobacco withdrawal symptoms, negative affect and cravings for cigarettes immediately following exercise during nine weeks of the treatment (8). Other studies confirm that exercise has an acute moderating effect on tobacco withdrawal and urges to smoke (9) and results in a reduction in post-smoking cessation weight gain (10). The five studies finding no effect of an exercise intervention on smoking abstinence had various methodological limitations; including, a small sample size and an inadequate smoking cessation programme or control condition (4).

In practice, the intensive exercise regimens adopted in previous studies of exercise and smoking cessation, focusing on supervised and structured vigorous exercise, are probably beyond the scope of many smoking cessation services, and are likely to be less cost-effective than interventions emphasising independent and 'lifestyle' type exercise (11). Furthermore, current physical activity recommendations encompass short, intermittent and daily bouts of moderate intensity exercise and encourage tailored regimens, including routine 'lifestyle' activities such as walking (12). In addition, there is some evidence to suggest that a counselling based intervention promoting lifestyle type exercise can result in comparable increases in exercise as for a more structured and supervised exercise intervention (13). Consequently, the present study adopted a more public health oriented approach than used in previous studies of 
exercise and smoking cessation through promoting exercise with exercise counselling alone. A randomized controlled trial was conducted to examine whether adding brief exercise counselling to a smoking cessation treatment programme including could increase rates of smoking abstinence and reduce post-cessation weight and body fat gain, tobacco withdrawal, and urges to smoke.

\section{METHODS}

\section{Participants}

Men and women aged 18 to 65 years, who had been smoking at least 10 cigarettes a day for at least three years, were motivated to stop smoking, and had not engaged in 30 minutes of moderate intensity exercise on five days a week, or 20 minutes of vigorous exercise on three days a week during the past three months (12), were recruited through newspaper advertisements or referral from their physician. Smokers who had a current psychiatric illness or substance misuse problem, were pregnant or planning a pregnancy, or who had a medical condition that would make compliance with the exercise regimen difficult (14) were excluded.

\section{Research design}

The study was a randomized controlled trial comparing two conditions: (i) a cognitive-behavioral and NRT smoking cessation programme plus brief exercise counselling ('exercise') and (ii) the same smoking cessation programme plus equal staff contact time ('control'). It was necessary to control for exposure time in this study in order to separate the effect of the exercise counselling from the effect of frequent contact with the investigator. On attendance at their first treatment session individual smokers were assigned to either the exercise or the control condition using a computer generated list of random numbers (Figure 1). Participants provided 
written consent and physicians were informed of their patients involvement in the study. The ethics committee of St. George's Hospital gave its' approval.

\section{Treatment regime}

\section{Smoking cessation programme}

All participants attended six individually-based weekly smoking cessation treatment sessions and a follow-up session two weeks after the final treatment. The first session took approximately 30 minutes. The other sessions lasted for 15-20 minutes. A cognitive-behavioural format was used incorporating self-monitoring, goal-setting, preparing for high risk situations, and coping with tobacco withdrawal $(15,16)$. Participants were required to stop smoking immediately prior to their second treatment session. Two Masters-level smoking cessation counsellors delivered the treatment programmes via standardised manuals.

\section{Nicotine replacement therapy}

NRT has been shown to be one of the most effective aids to smoking cessation (3) and is routinely recommended by stop smoking services (17). Compliance tends to be higher with transdermal nicotine patches compared to other forms of NRT, and patches have been shown to be as effective for increasing smoking abstinence as other NRT products (18). Therefore, in the current study all smokers were advised to use 15mg 16- hour patches $(19,20)$, following their final cigarette, on a daily basis, throughout the treatment programme $(3,21)$.

\section{Exercise condition}

The intention was to use an exercise intervention which could be readily integrated into existing behavioural smoking cessation treatments and which could be 
implemented by personnel who were not exercise specialists. At the first treatment session only those in the exercise condition received approximately five minutes of cognitive-behavioural exercise counselling incorporating decision balance sheets, goal-setting, relapse prevention planning, and self-monitoring (22). The investigators received two days of training in exercise counselling (23). Participants were encouraged to use exercise as a self-control strategy for reducing cigarette cravings and withdrawal (24). From session two onwards exercise levels in the previous week were briefly reviewed (one-two minutes) and encouragement was given towards maintaining any increases in exercise.

Participants were advised to progress over the seven weeks of the trial towards 30 minutes of 'lifestyle' (22) or more structured exercise, on at least five days a week, in bouts lasting at least five minutes (12). Short bouts of exercise have been found to be effective for improving body composition (25) and for reducing tobacco withdrawal symptoms and urges to smoke (9).

In contrast to the previous study showing an effect of exercise on smoking abstinence (7) the present study promoted moderate as well as vigorous intensity exercise. Moderate intensity exercise has been shown to provide both greater psychological benefits (26) and higher adherence rates (27) when compared with vigorous intensity exercise; and has been shown to reduce tobacco withdrawal and urges to smoke (9). At each session a resting radial pulse was used to prescribe at least a moderate intensity of exercise corresponding to $40 \%$ of heart-rate reserve (28), and the Borg 6-20 scale was used to recommend a Rating of Perceived Exertion of at least 'fairly light' (29).

\section{Control condition}

During the first treatment session only those in the control condition received 
approximately five minutes of heath education advice relating to healthy eating, fat and salt intake, alcohol consumption and stress management, derived from literature produced by the British Heart Foundation. A similar programme has been shown to be acceptable in previous trials of exercise for smoking cessation $(5,7)$. As a substitute for the self-monitoring used in the exercise group those in the control group monitored situations in which they found it difficult not to smoke, and from session two onwards these reports were briefly (duration of approximatelyone-_two minutes) reviewed each week so as to provide equal contact time as for the exercise condition.

\section{Measures}

At the first visit information was gathered relating to age, gender, marital status, employment, occupation, ethnicity, years of education, stage of change for exercise (30), cigarettes smoked per day, nicotine dependence (31), and expired air carbon monoxide (CO) level using a Bedfont Smokerlyzer. All baseline measures were administered before the smokers were assigned to a treatment group.

The main outcome analysis and power calculations for the study were based on continuous smoking abstinence from the quit date through six weeks. While many smokers relapse to smoking after that time, and 12 months of follow up are often considered necessary to demonstrate efficacy, the six-week period is used by the US Food and Drug Administration when deciding on efficacy of pharmaceutical smoking cessation aids. Furthermore, in the absence of short-term follow-up studies it would have been premature to mount the size of study required to test efficacy for 12 months.

Following one week of smoking abstinence, and then on a weekly basis, smokers reported whether they had smoked since the previous visit (32). Smoking abstinence was verified with expired air CO concentration (cut-off, 10ppm). If a participant 
smoked even a single puff, or if they failed to attend a treatment or follow-up session and an appointment could not be made within 48 hours, they were excluded from the programme and were recorded as having lapsed. Those smokers who were excluded from the study were offered further smoking cessation support from another clinic, although their progress was not monitored.

At each visit following the quit day participants reported their type and dose of NRT, and reported their weekly urges to smoke on six-point scales in response to three items (33): 'How difficult has it been to stop yourself smoking in the past week?' $(0=$ not at all difficult to $5=$ extremely difficult $)$, 'How much of the time have you felt the urge to smoke in the past week?' $(0=$ not at all to $5=$ all the time), and 'How strong have the urges been?' ( $0=$ no urges to $5=$ extremely strong). Urges to smoke are generally strongest in the first week of abstinence (34). Therefore on each day during the first week of abstinence participants responded on a six-point scale to the question 'How strong is your desire to smoke?' $(35,0=$ no urges to $5=$ extremely strong).

Tobacco withdrawal symptoms were reported by the participants at the first visit and then at each visit following the quit day using the Mood and Physical Symptoms Scale (MPSS, 33, 35). The items included were irritable, restless, tense, anxious, happy, stressed, depressed, hungry, poor concentration, and poor sleep. For each item participants were asked to rate 'How you have been feeling over the past week' on a five-point scale ( $1=$ not at all to $5=$ extremely).

Levels of physical activity were self-reported at baseline and after one, four and six weeks of smoking abstinence using the Seven Day Physical Activity Recall Questionnaire (36). During a structured interview participants recalled episodes of physical activity for each day during the previous week. The hours spent in five 
categories of activity intensity were multiplied by the assigned Metabolic Equivalent Task (MET) value for each category; where sleep=1 MET, light=1.5 METS, moderate $=4$ METS, hard $=6$ METS, and very hard=10 METS. The resulting weekly MET score was divided by seven to give a mean daily MET score. Hours of at least moderate intensity activity, hours of vigorous intensity activity ('hard' plus 'very hard') and days with 30 minutes or more of at least moderate intensity activity were also determined.

Participants in the exercise group only were asked to record their heart-rate by radial pulse immediately following a bout of at least moderate intensity exercise on each day of the week. For each individual a mean post-exercise heart-rate was computed for one, four and six weeks of smoking abstinence. Using the heart-rate reserve method for deriving heart-rate ranges (28), each participants weekly heartrates mean was then categorised as corresponding to a 'light', 'moderate' or 'vigorous' intensity of exercise.

At baseline clothed weight and height, without shoes, were measured on calibrated scales to the nearest $0.25 \mathrm{~kg}$ and $0.5 \mathrm{~cm}$, respectively. Percentage body fat was estimated using a Maltron $50 \mathrm{khz}$ hand-to-foot bioelectrical impedance analysis device (37). Further measures of weight and body fat were taken after one, four and six weeks of smoking abstinence.

Participants expectations for the treatment regime were reported at the end of visits one and two as adapted from standard protocols $(38,39)$. Patients were asked to indicate their strength of agreement on a five-point scale (1=strongly disagree to 5 $=$ strongly agree) with three statements: (i) 'I believe that taking part in this programme will increase my chances of stopping smoking for good', (ii) 'I expect to enjoy taking part in this programme', and (iii) 'I would recommend this programme to 
a friend'.

\section{Statistical analysis}

Based on the findings of Marcus and colleagues (7) it was estimated that a sample of 300 smokers would yield $80 \%$ power (at $\mathrm{P}<.05$, two-tailed) at six weeks of continuous smoking abstinence, to detect a difference in abstinence between the treatment and control groups of $45 \%$ versus $30 \%$. Unless otherwise stated analyses were conducted on an intention-to-treat basis according to random assignment at visit one, with twotailed tests, and using SPSS version 10. The baseline characteristics of the two groups were compared using analysis of variance (ANOVA) and chi-squared $\left(\chi^{2}\right)$ test.

ANOVA and $\chi 2$ test were used to analyse differences in smoking abstinence, exercise levels, use of NRT, withdrawal symptoms, urges to smoke, weight, body mass index (BMI) and estimated body fat by treatment condition. Regression analysis and correlation were used to examine baseline differences between the treatment groups as co-variates in the analysis for smoking abstinence, tobacco withdrawal and exercise levels. Regression analysis was used to examine whether exercise levels at baseline or adherence to the exercise intervention predicted smoking abstinence.

\section{RESULTS}

\section{Participants}

Of 696 smokers screened by telephone 200 were excluded from the study, 197 decided not to participate, and 299 were randomized to the study (Figure 1). Baseline characteristics are presented in Table 1. The sample was predominantly female $(62.9 \%, 188 / 299)$, white $(87.9 \%, 263 / 299)$, employed $(67.2 \%, 201 / 299)$, and in nonprofessional/managerial occupations $(55.5 \%, 166 / 299)$. Around half the sample was 
married $(52.5 \%, 157 / 299)$. The vast majority of the sample reported being at a stage of change for exercise of 'contemplation' ('I am planning to become more active in the next six months', 72.2\%, 216/299). L, Whilst whilst 27.8\% (83/299) reported being at a pre-contemplation stage ('I have no plan to become more active in the next six months'). Due to the recruitment criteria none of the participants reported being at a stage of change for exercise of 'preparation', 'action' or 'maintenance'. At baseline the exercise group was found to be younger $(\mathrm{F}=5.2, \mathrm{P}=.02)$, was more likely to report being in a 'contemplation' rather than a 'pre-contemplation' stage of change for exercise $(\mathrm{F}=7.0, \mathrm{P}=.0009$; exercise group: contemplation $=77.9 \%, 120 / 154$; control group: contemplation $=66.2 \%, 96 / 145)$ and gave higher ratings for the MPSS items of restlessness and poor sleep when compared with the controls $(\mathrm{F}=13.4, \mathrm{P}<.001 ; \mathrm{F}=5.6$, $\mathrm{P}=.02$, respectively). There were no other baseline differences between the two groups.

\section{Adherence to treatment regime}

The data for adherence to the exercise regimen (Table 2) was analysed exclusively for those abstinent from smoking through to the final visit, using ANOVAs with planned comparisons unless stated otherwise. Reports of hours of moderate or vigorous exercise were significantly higher for the exercise group than for the controls (all at $\mathrm{P}$ $<.001)$ for baseline compared to one week $(F=31.0)$, four weeks $(F=14.1)$, and six weeks of abstinence $(\mathrm{F}=17.9)$.

The exercise group reported significantly more hours of vigorous intensity exercise than the controls after four weeks of smoking abstinence (Mann-Whitney, $Z=$ 2.7, $\mathrm{P}<.01$ ), but not after one or six weeks of abstinence. Total METS per day were higher for the exercise group than for the controls for baseline compared to one week 
$(\mathrm{F}=21.0, \mathrm{P}<.001)$, four weeks $(\mathrm{F}=19.9, \mathrm{P}<.001)$, and six weeks of abstinence $(\mathrm{F}=7.1$, $\mathrm{P}=.009)$. The exercise group reported more days with 30 minutes of moderate or vigorous exercise for baseline compared with one, four and six weeks of abstinence, $(\mathrm{F}=35.1 ; \mathrm{F}=22.0 ; \mathrm{F}=18.8$, respectively, all at $\mathrm{P}<.001)$. By the final visit $23.0 \%(14 / 61)$ of the exercise group and $7.1 \%(4 / 56)$ of the control group reported at least five days of 30 minutes of moderate or vigorous exercise.

Differences at baseline between the treatment groups were examined as covariates in the analysis for changes in reports of hours of exercise between baseline and one week of smoking abstinence. Regression analysis showed that being in a more advanced stage of change for exercise predicted reports of a greater increase in exercise $(\mathrm{Beta}=0.186, \mathrm{P}=0.009)$. Other baseline differences were not found to be related to exercise adherence at this time. Using a forced entry multiple regression analysis, controlling for the variable stage of change for exercise, treatment group remained significant as a predictor of increases in hours of exercise at week one.

Following one, four and six weeks of smoking abstinence the percentage of participants in the exercise group having a mean post-exercise heart-rate corresponding to at least a moderate intensity were $61.7 \%(50 / 81), 68.9 \%(31 / 45)$, and $82.3 \%$ (28/34), respectively. At both baseline and at the final visit the most frequently reported mode of exercise was walking, both for the exercise group $(50.7 \%(99 / 195)$; $73.3 \%$ (33/45), respectively) and for the controls (53.1\% (51/96); $52.9 \%(18 / 34)$, respectively).

Following one and six weeks of smoking abstinence the vast majority of participants reported using NRT $(90 \%, 162 / 180 ; 67 \%, 75 / 117$, respectively). A chisquared test showed no significant differences in NRT use for the exercise group versus the controls at any measurement point. Following one week of abstinence $87 \%$ 
$(141 / 162)$ of NRT users reported using the transdermal nicotine patch. Of these $64.5 \%$

(91/141) reported using the $15 \mathrm{mg}$ patch, $14.9 \%(21 / 141)$ the $21 \mathrm{mg}$ patch, and $20.6 \%$

(29/141) the 10mg patch. ANOVAs revealed no difference in expectations for the treatment regimen between the exercise and control groups at either visits one or two.

\section{Smoking outcome}

Six weeks following their quit day $39.6 \%(61 / 154)$ of exercise participants and $38.6 \%$ (56/145) of controls were continually abstinent from smoking (Table 3a, 95\%

Confidence Interval, -10.1 to 12.1 for differences between groups). A $\chi^{2}$ test did not reveal any significant differences between the treatment groups for rates of continuous abstinence at any measurement point. When examining females and males separately a $\chi 2$ test did not show any significant differences between the treatment groups for smoking abstinence at any measurement point. For females the six week abstinence rates for the exercise and control groups were $43.8 \%$ (42/96) versus $39.1 \%(36 / 92)$, for males it was $32.8 \%(19 / 58)$ versus $37.7 \%(20 / 53)$.

Attendance at the quit day visit was slightly, though not significantly, higher for the control group than for the exercise group (89\% (129/145) versus $83.1 \%(128 / 154)$, respectively); thus reducing the chance of finding a significant effect for the exercise intervention. When examining smoking abstinence rates exclusively for those attending their quit day (Table 3b) a $\chi^{2}$ test revealed higher rates of abstinence for the exercise group compared with the controls after both one and two weeks of abstinence $(\chi=5.2, \mathrm{P}=.03 ; \chi=4.9, \mathrm{P}=.03$, respectively), but not at the other measurement points. Regression analysis showed that neither reports of levels of exercise at baseline nor stage of change for exercise predicted attendance at the quit day session. This was the case both for the sample as a whole and also when looking at the exercise group 
alone.

Using regression analysis, for the sample as whole, neither reports of hours of moderate or vigorous intensity exercise at baseline nor reports of increases in hours of this exercise between baseline and one week of smoking abstinence predicted smoking abstinence at six weeks $(\mathrm{OR}=.93, \mathrm{P}=.46 ; \mathrm{OR}=.98, \mathrm{P}=.79$, respectively). Nor did reports of hours of vigorous exercise at baseline or increases in vigorous exercise between baseline and one week of abstinence predict smoking abstinence at six weeks $(\mathrm{OR}=.65, \mathrm{P}=.11 ; \mathrm{OR}=.14, \mathrm{P}=.14$, respectively). When looking exclusively at the exercise group, again using regression analysis, smoking abstinence at six weeks was not predicted either by reports of increases in hours of moderate or vigorous exercise between baseline and one week $(\mathrm{OR}=0.99, \mathrm{P}=.89)$, nor by reports of increases in hours of vigorous exercise between baseline and one week $(\mathrm{OR}=1.09, \mathrm{P}=.50)$.

Differences at baseline between the treatment conditions were examined as covariates in the analysis for smoking abstinence at six weeks. Logistic regression showed that greater age predicted higher rates of smoking abstinence, both for the intention-to-treat analysis and for the analysis using only those attending the quit day $(\mathrm{OR}=1.02, \mathrm{P}=.03$, in both cases $)$. Baseline ratings of restlessness or poor sleep did not predict rates of smoking abstinence. Using a forced entry multiple logistic regression analysis, controlling for the variable age, treatment group remained non-significant as a predictor of smoking abstinence at six weeks, both for the sample as a whole and for those attending the quit day.

\section{Tobacco withdrawal and urges to smoke}

Separate treatment group by time ANOVAs were conducted between baseline and each further measurement point for each MPSS item, including all smokers abstinent from smoking at each measurement point. For baseline compared to one week of 
smoking abstinence significant group by time interactions were found (Table 4a) for the items of tension $(\mathrm{F}=5.0, \mathrm{P}=.03)$, anxiety $(\mathrm{F}=6.5, \mathrm{P}=.01)$, happiness $(\mathrm{F}=5.3, \mathrm{P}=.03)$, and stress $(\mathrm{F}=3.3, \mathrm{P}=.04$, one-tailed $)$. There were significant interactions for irritability through two weeks of abstinence relative to baseline $(\mathrm{F}=4.7, \mathrm{P}=.03)$, and for restlessness through three weeks of abstinence relative to baseline $(\mathrm{F}=4.4, \mathrm{P}=.04$; Table 4b). For the happiness item ratings were higher for the exercise group than for the controls. In all other cases ratings were lower for the exercise group relative to the controls. There was no evidence of group differences for the items of depression, hunger, poor concentration, or poor sleep. For those abstinent through to the final visit ANOVAs, using planned comparisons, did not reveal any significant treatment group by time interactions for baseline compared to one week of abstinence for any of the withdrawal items. Using regression analysis there was no evidence for baseline differences between the treatment groups acting as confounders in the analyses for the withdrawal items.

ANOVAs did not reveal any between group differences for the weekly urge to smoke items or for a composite item combining the three separate urges to smoke items. This was the case both for those remaining abstinent from smoking at each measurement point and for those remaining abstinent throughout. For daily urges to smoke during the first week of abstinence ANOVAs, using planned comparisons, did not show any group differences, either for those remaining abstinent for one week, or for those abstinent throughout.

\section{Weight and body fat gain}

ANOVAs did not show any significant group by time differences between baseline and six weeks of smoking abstinence for weight, BMI, or estimate of percentage body fat. For those remaining abstinent throughout, in the exercise and control groups, the 
mean (SD) weight gain $(\mathrm{kg})$ was 1.8 (1.9) and 2.0 (1.9), respectively; the mean increase in BMI was $0.6(0.7)$ and $0.7(0.7)$, respectively; and the mean increase in estimated percentage body fat was 0.7 (4.5) and 0.5 (5.2), respectively.

\section{CONCLUSIONS AND DISCUSSION}

On an intention-to-treat basis, including a brief exercise counselling intervention in a smoking cessation treatment package resulted in only modest and non-significant increases in smoking abstinence in comparison to an equal contact control group. Nor was the amount of increase in exercise levels found to be related to smoking abstinence. However, the chance of detecting a significant difference in abstinence rates between the exercise and control groups was reduced by the exercise group having slightly lower attendance rates at the quit day visit relative to the controls. There were no differences in reports of expectations for the treatment regime between the treatment groups. Therefore the difference in attendance rates at the quit day was probably by chance. It is possible that the lower rates of attendance at the quit day session for the exercise group versus the controls was due to some of the smokers assigned to the exercise group being unwilling to engage in the exercise regimen, although neither baseline levels of exercise nor stage of change for exercise were shown to be related to quit day attendance. Future studies of exercise and smoking cessation could examine this issue further through exploring the smokers' reasons for not attending their quit day visit. The finding that for those attending the quit day visit there were significantly higher abstinence rates for the exercise group versus the controls up to the first two weeks of abstinence indicates that exercise counselling may have some potential as an aid for smoking cessation.

The difference in findings between the present study and the previous study (7) showing a positive effect of an exercise intervention on smoking cessation is likely to 
be partly a result of differences in the exercise intervention between the two studies. The previous study, by Marcus and colleagues (7), employed a 12 12-week 36 session supervised exercise programme. The current study used a more public health oriented intervention requiring only five minutes of exercise counselling plus one-two minutes per week of further support over seven weeks. Consequently, the smokers in the present study were engaged in a shorter duration of exercise programme and also reported lower exercise intensities compared with the Marcus study. In the current study only $16.4 \%(10 / 61)$ of the participants in the exercise group reported any vigorous intensity at the final treatment. , Whereas whereas in the Marcus study vigorous intensity exercise was adopted throughout. Reports of increases in hours of exercise after stopping smoking in the present study were comparable to increases observed in the Marcus study. However, exercise data in the current study was based exclusively on self-reports and it is possible that those receiving the exercise intervention were biased towards overestimation of their exercise levels. Future studies of this type need to include more objective monitoring of physical activity levels; for example, through use of heart-rate monitoring or accelerometry $(40,41)$.

Further studies are needed to determine the minimum levels of exercise and exercise intervention required to result in significant long-term increases in smoking abstinence rates; for example, receiving professional support is likely to be an important factor in increasing exercise adherence (42). Additionally, there is some evidence to suggest that abstinent smokers tend to overestimate exercise intensities (9), and therefore may need supervision with gauging these intensities. Therefore, an intervention combining exercise counselling with some supervised exercise is likely to increase exercise levels beyond those reported in the present study. In addition, studies are needed to compare the effect of different doses of exercise on smoking 
abstinence within the same study.

The present study is the first to combine an exercise programme with a smoking cessation treatment which incorporates NRT. Therefore the difference in findings between the present study and the Marcus study may also be partly due to the inclusion of NRT in the current study. NRT has previously been shown to approximately double smoking abstinence rates (3), and the overall abstinence rate reported at six weeks in the present study $(39.1 \%)$ was approximately double that found for the exercise group in the Marcus study at eight weeks (19.4\%). It is feasible that exercise and NRT target similar mechanisms as aids to smoking cessation, and therefore do not have a marked additive effect on abstinence rates. For example, nicotine and exercise may have some common physiological effects, such as increases in the release of adrenocorticoids and opioids $(43,44)$; and some shared psychological effects in terms of reducing tobacco withdrawal and urges to smoke $(9,45)$. Studies are needed to compare the effects on smoking abstinence of combined nicotine and exercise interventions with nicotine and exercise alone. Studies combining exercise with NRT may need to consider larger sample sizes in order to detect any modest effects of an exercise intervention. Additionally, exercise needs to be examined as an aid to smoking cessation for those who are contraindicated for NRT or who prefer not to use it; for example, amongst pregnant smokers.

Although reports of exercise levels were higher for the exercise group than for the controls, following six weeks of smoking abstinence the exercise group gained only $0.2 \mathrm{~kg}$ less in body weight than the controls, a difference which did not reach statistical significance. This finding is in contrast with that of Marcus and colleagues (7) who observed that, following eight weeks of smoking cessation, those receiving an intensive supervised exercise programme gained $2.4 \mathrm{~kg}$ less body weight than an equal 
contact control group. The lack of an effect on weight gain in the present study may be partly due to the lower intensities of exercise achieved in this study compared with the Marcus study. It may also have been related to the inclusion of NRT in the present study. Post-cessation weight gain is likely to be less pronounced when NRT is used (46). Therefore, at the outset the potential for exercise to moderate weight gain was reduced. Further studies are needed to determine the effect of varying doses of exercise on post-smoking cessation weight and body fat gain, both during NRT use and following discontinuation of NRT. An effective intervention for reducing postsmoking cessation weight gain might attract smokers who fear weight gain (47), and could reduce relapses resulting from weight gain (48).

This is the first study to show a more long-term, as opposed to acute, effect of exercise on some psychological symptoms following smoking cessation. Previously, the only psychological symptoms which that have been shown to reliably predict rates of smoking abstinence are depression and urges to smoke (49). Contrary to the findings of acute studies $(8,9)$ the exercise intervention in the present study did not affect either of these variables. Evidence for the moderating effect of exercise on some psychological symptoms may increase the acceptability of exercise as an aid to smoking cessation. However, the reported effects of the exercise intervention on psychological symptoms were modest and it is possible that they were partly the result of attention placebo influences, whereby those in the exercise group become persuaded of the benefits of exercise and therefore report more psychological benefits than the control group. Objective validation of reports of physical activity would help to resolve this issue. In addition, studies are required in order to determine the effects of varying doses of exercise on tobacco withdrawal and on urges to smoke.

In summary, adding brief exercise counselling to a smoking cessation programme 
combining behavioural support and NRT resulted in only modest and non-significant increases in smoking abstinence. There was no evidence for the inclusion of exercise counselling resulting in a significant reduction in weight gain. The exercise intervention was shown to increase exercise levels and to moderate some psychological symptoms during the first few weeks of smoking abstinence. Further studies are needed to determine the effect of varying doses of exercise on smoking abstinence, and to examine the interaction between exercise and pharmaceutical aids to smoking cessation.

\section{Acknowledgements}

This study was supported through grant CE1198/0101 from the Cancer Research Campaign (now Cancer Research UK) to the authors.

\section{REFERENCES}

1. Peto R., Lopez A. D., Boreham J., Thun, M., Heath C Jr. \& Doll R. (1996)

Mortality from smoking worldwide. British Medical Bulletin, 52, 12-21.

2. Doll R., Peto R., Wheatley K., Gray R. \& Sutherland I. (1994) Mortality in relation to smoking: 40 years' observations on male British doctors. British Medical Journal, 309, 901-911.

3. Silagy C., Mant D., Fowler G. \& Lancaster T. (2000) Nicotine replacement therapy for smoking cessation. Cochrane Database of Systematic Reviews. The Cochrane Collaboration, 3. Oxford: Update Software. 
4. Ussher M. H., West R., Taylor A. H. \& McEwen A. (2000) Exercise interventions in smoking cessation. Cochrane Database of Systematic Reviews, The Cochrane Collaboration, 3. Oxford: Update Software.

5. Marcus B., Albrecht A. E. \& Niaura R. S. (1995) Exercise enhances the maintenance of smoking cessation in women. Addictive Behaviors, 20, 87-92.

6. Martin J. E., Kalfas K. J., \& Patten C. A. (1997) Prospective evaluation of three smoking interventions in 205 recovering alcoholics: One-year results of project SCRAP-Tobacco. Journal of Consulting \& Clinical Psychology, 65, 190-194.

7. Marcus B. H., Albrecht R. N., King T. K., Parisi A. F., Pinto B. M., Roberts M., Niaura R. S. \& Abrams D. B. (1999) The efficacy of exercise as an aid for smoking cessation in women: a randomized controlled trial. Archives of Internal Medicine, $159,1229-1234$.

8. Bock B. C., Marcus B. H., King T. K., Borrelli B. \& Roberts M.R. (1999) Exercise effects on withdrawal and mood among women attempting smoking cessation. Addictive Behaviors, 24, 399-410.

9. Ussher M., Nunziata P., Cropley M. \& West R. (2001) Effect of a short bout of exercise on tobacco withdrawal symptoms and desire to smoke. Psychopharmacology, $158,66-72$.

10. Kawachi I., Troisi R. J. , Rotnitzky A. G., Coakley E. H., Colditz M. S. \& Colditz 
M. D. (1996) Can physical activity minimise weight gain in women after smoking cessation? American Journal of Public Health, 86, 999-1004.

11. Sevick M. A., Dunn A. L., Morrow M. S., Marcus B. H., Chen G. J., \& Blair S. N. (2000) Cost-effectiveness of lifestyle and structured exercise interventions in sedentary adults: results of project ACTIVE. American Journal of Preventive Medicine, 19, 1-8.

12. Pate P. R., Pratt M., Blair S. N., Haskell W. L., Macera C. A., Bouchard C., Buchner D., Ettinger W. E., Heath G. W., King A.C., Krishka A., Leon A. S., Marcus B. H., Morris J., Paffenbarger R. S., Patrick K., Pollock M. L., Rippe J. M., Sallis J. \& Wilmore J. H. (1995) Physical Activity and public health: A recommendation from the Centers for Disease Control and Prevention and the American College of Sports Medicine. Journal of the American Medical Association, 273, 402-407.

13. Dunn A. L., Garcia M. E., Marcus B. H., Kampert J. B., Kohl H. W., \& Blair S. N. (1998) Six-month physical activity and fitness changes in Project Active, a randomized trial. Medicine and Science in Sports and Exercise, 30, 1076-1083.

14. Thomas S., Reading J. \& Shephard R. J. (1992) Revision of the Physical Activity Readiness Questionnaire (PAR-Q). Canadian Journal of Sport Science, 17, 338-345.

15. Jorenby D. E., Smith S. S., Fiore M. C., Hurt R. D., Offord K. P., Crogham, I. T., Hays J. T., Lewis S. F. \& Baker T. B. (1995) Varying nicotine patch dose and type of smoking cessation counseling. Journal of the American Medical Association, 274, 
16. West R. \& Willis N. (1998) Double-blind placebo controlled trial of dextrose tablets and nicotine patch in smoking cessation. Psychopharmacology, 136, 201-204.

17. West R., McNeill A. \& Raw M. (2000) Smoking cessation guidelines for health professionals: an update. Thorax, 55, 987-999.

18. Hajek P., West R., Foulds J., Nilsson F., Burrows S. \& Meadows A. (1999) Randomised comparative trial of nicotine gum, transdermal nicotine patch, nasal spray and inhaler. Archives of Internal Medicine, 159, 2033-2038.

19. Dale L., Hurt R., Offord K. \& Lawson G. (1995) High-dose nicotine patch therapy: percentage of replacement and smoking cessation. Journal of the American Medical Association, 274, 1353-1358.

20. Hughes J., Lesmes G., Hatsukami D., Higgins S., Bickel W., Richmond R., Lichtenstein E., Jorenby D. E., Broughton J. O., Fortmann S. P., Leischow S. J., McKenna J. P. Rennard S. I., Wadland W. C. \& Hentley S. A. (1999) Are higher doses of nicotine replacement more effective for smoking cessation? Nicotine and Tobacco Research, 1, 169-174.

21. Tonnesen P., Paoletti P., Gustavsson G, Russel M. A., Scracci R., Gulsvik A., Rijcken B. \& Sawe U. (1999) Higher dosage nicotine patches increase one-year smoking cessation rates: results from the European CEASE trial. European 
Respiratory Journal, 13, 238-246.

22. Dunn A. L., Marcus B. H., Kampert J. B., Garcia M. E., Kohl H. W. \& Blair S. N. (1999) Comparison of lifestyle and structured interventions to increase physical activity and cardiovascular fitness: a randomized trial. Journal of the American Medical Association, 281, 327-334.

23. Health Education Authority. (1994) Helping people change: Health promotion in primary health care. London: Health Education Authority.

24. Marlatt G. A. \& Gordon J. R. (1985) Relapse prevention: Maintenance Strategies in the Treatment of Addictive Behaviors. New York: Guildford.

25. Coleman K. J., Raynor H. R., Mueller D. M., Cerny F. J., Dorn J. M. \& Epstein L. H. (1999) Providing sedentary adults with choices for meeting their walking goals. Preventive Medicine, 28, 510-519.

26. Byrne A. \& Byrne D. G. (1993) The effect of exercise on depression, anxiety and other mood states: a review. Journal of Psychosomatic Research. 37, 565-574.

27. Sallis J. F., Haskell W. L., Fortmann S. P., Vranizan K. M., Taylor C. B. \& Solomon D.S. (1986) Predictors of adoption and maintenance of physical activity in a community sample. Preventive Medicine, 15, 331-341.

28. American College of Sports Medicine (2000) American College of Sports 
Medicine Guidelines for Exercise Testing and Prescription. 6th ed. Philadelphia: Williams \& Wilkins.

29. Borg G. A. V. (1998) Borg's Perceived Exertion and Pain Scales. Champaign, Illinois: Human Kinetics.

30. Marcus B. H., Rossi J. S., Selby V. C., Niaura R. S., Abrams D. B. (1992) The stages and processes of exercise adoption and maintenance in a worksite sample. Health Psychology, 11, 386-395.

31. Heatherton T., Kozlowski L., Frecker T. \& Fagerström K. (1991) The Fagerström Test for Nicotine Dependence: a revision of the Fagerström Tolerance Questionnaire. Addiction, 86, 1119-1127.

32. West R., Edwards M. \& Hajek P. (1998) A randomised controlled trial of a buddy system to improve success at giving up smoking in general practice. Addiction, 93, 1007-1011.

33. West R., Hajek P. \& Belcher M. (1989) Severity of withdrawal symptoms as a predictor of outcome of an attempt to quit smoking. Psychological Medicine, 19, 981-985.

34. Shiffman S. \& Jarvik M. E. (1976) Smoking withdrawal symptoms in two weeks of abstinence. Psychopharmacology, 50, 35-39. 
35. West R. \& Russell M. (1985) Pre-abstinence smoke intake and smoking motivation as predictors of severity of cigarette withdrawal symptoms. Psychopharmacology, 87, 334-336.

36. Blair S. N., Haskell W. L., Ho P., Paffenbarger P., Vranizan K. M., Farquhar J. W. \& Wood P.D. (1985) Assessment of habitual physical activity by seven-day recall in a community survey and controlled experiments. American Journal of Epidemiology, $122,794-804$.

37. Roubenoff R. (1996) Applications of bioelectrical impedance analysis for body composition to epidemiologic studies. American Journal of Clinical Nutrition, 64, S459-S462.

38. Bootzin R. R. \& Lick J. R. (1979) Expectancies in therapy research: interpretative artefact or mediating mechanism? Journal of Consulting and Clinical Psychology, 47, $852-855$

39. Lick J. \& Bootzin R. (1975) Expectancy factors in the treatment of fear: methodological and theoretical issues. Psychological Bulletin, 82, 917-931.

40. Bassett D. R. Jr, Ainsworth B. E., Swartz A. M., Strath S. J., O'Brien W. L., King G. A. (2000) Validity of four motion sensors in measuring moderate intensity physical activity. Medicine \& Science in Sports \& Exercise, 32, S471-480.

41. Strath S. J., Swartz A. M., Bassett D. R. Jr., O'Brien W. L., King G. A., Ainsworth 
B. E. (2000) Evaluation of heart rate as a method for assessing moderate intensity physical activity. Medicine \& Science in Sports \& Exercise, 32, S465-470.

42. Hillsdon M., Thorogood M., Anstiss T. \& Morris J. (1995) Randomised controlled trials of physical activity in free living populations: A review. Journal of Epidemiology \& Community Health, 49, 448-453.

43. Morgan W. P. ed. (1997) Physical Activity and Mental Health. London: Taylor \& Francis.

44. Royal College of Physicians. (2000) Nicotine Addiction in Britain: A report of the Tobacco Advisory Group of the Royal College of Physicians. London: Royal College of Physicians of London.

45. West R. \& Shiffman S. (2001) Effect of oral nicotine dosing forms on cigarette withdrawal symptoms and craving: a systematic review. Psychopharmacology, $155: 115-122$.

46. Jorenby D. E., Hatsukami D. K., Smith S. S, Fiore M. C., Allen A., Jensen J. \& Baker T.B. (1996) Characterization of tobacco withdrawal symptoms: transdermal nicotine reduces hunger and weight gain. Psychopharmacology, 128, 130-138.

47. Pomerleau C. S., Zucker A. N. \& Stewart A. J. (2001) Characterizing concerns about post-cessation weight gain: results from a national survey of women smokers. Nicotine Tobacco Research, 3, 51-60. 
48. Borrelli B. \& Mermelstein R. (1998) The role of weight concern and self-efficacy in smoking cessation and weight gain among smokers in a clinic-based cessation program. Addictive Behaviors, 23, 609-622.

49. Shiffman S., West R. \& Gilbert D. G. (2002) The assessment of tobacco craving and withdrawal in smoking cessation trials. Nicotine Tobacco Research, in press. 
Table 1. Baseline characteristics of study groups

\begin{tabular}{|c|c|c|c|}
\hline Characteristics & \multicolumn{3}{|c|}{ Exercise Group (N=154) Mean (SD) Control } \\
\hline Group $(\mathrm{N}=145)$ Mean (SD) & \multicolumn{3}{|c|}{$\mathrm{P}^{*}$} \\
\hline Age, y & $41.5(11.1)$ & $44.4(11.1)$ & 0.02 \\
\hline Education, $\mathrm{y}$ & $12.8(3.1)$ & $12.8(3.0)$ & 0.89 \\
\hline Weight, kg & $\$ 71.8(14.4)$ & $\S 72.5(15.4)$ & 0.72 \\
\hline Body Mass Index, $\mathrm{kg} / \mathrm{m}^{2}$ & $\$ 25.4(4.5)$ & $\$ 25.8(4.8)$ & 0.46 \\
\hline Body fat, $\%$ & $\S 27.9(9.2)$ & $\S 28.7(9.2)$ & 0.44 \\
\hline \multicolumn{3}{|c|}{ Fagerström Test for Nicotine Dependence score (range 0-10) } & 5.4 \\
\hline & $5.8(2.0)$ & 0.11 & \\
\hline Smoking rate, cigarettes/d & $21.6(8.8)$ & $22.3(9.2)$ & 0.53 \\
\hline Carbon monoxide level, ppm & $21.1(1.5)$ & $22.1(10.2)$ & 0.50 \\
\hline Weekly hours of exercise & $1.1(1.5)$ & $0.9(1.0)$ & 0.28 \\
\hline Daily METs & $32.7(1.4)$ & $32.6(0.7)$ & 0.47 \\
\hline
\end{tabular}

$\S$ Data missing for one participant $\S \S$ Data missing for two participants 
Table 2. Exercise adherence for those remaining abstinent from smoking throughout

\begin{tabular}{|l|l|} 
Exercise variable & Exercise group $n=61$ Mean $(\mathrm{SD})$ \\
& Control group $\mathrm{n}=56$ Mean $(\mathrm{SD})$
\end{tabular}

Hours of moderate or vigorous exercise in the previous week *

\begin{tabular}{|c|c|c|}
\hline$*$ & $*$ & a. $0.9(0.9)$ b. $2.6(1.7)$ c. $3.0(2.4)$ \\
\hline d. $3.0(2.4)$ & & a. $0.9(0.9)$ b. $0.9(1.2)$ c. $1.2(1.6)$ \\
\hline
\end{tabular}

Hours of vigorous exercise in the previous week

a. $0.1(0.4)$ b. $0.2(0.4)$ c. $0.3(0.9)$

d. $0.2(0.7)$

a. $0.1(0.1)$ b. $0.1(0.5)$ c. $0.1(0.2)$

d. $0.1(0.5)$

Daily METs

**

a. $32.5(0.8)$ b. $33.2(1.1)$ c. 33.4

(1.4) d. $33.4(2.2)$

a. $32.6(0.6)$ b. $32.5(0.8)$ c. 32.6

$(0.8)$ d. $32.7(0.8)$

\begin{tabular}{|l|l} 
Days with 30 minutes of moderate or vigorous exercise $*$ & in the
\end{tabular} previous week

$2.5(2.2)$ c. $2.4(2.3)$ d. $2.6(2.2)$

a. $0.7(0.9)$ b. $0.7(1.2)$ c. $0.7(2.1)$

d. $1.9(2.1)$

a.: baseline; b., c., d.: after one, four and six week of smoking abstinence,

respectively. Significant difference relative to baseline for exercise/control

comparison at $\mathrm{P}<.001 *$ and at $\mathrm{P}<.05 * *$ 
Table 3. Continual smoking abstinence by group

(*for differences between groups by $\chi^{2}$ )

Table 3a. On intention-to-treat basis (No./\%)

\begin{tabular}{|l|l|l|l|}
\hline Weeks of abstinence & Exercise group $(\mathrm{n}=154)$ & Control \\
\hline \multicolumn{4}{|l|}{$\mathrm{P}^{*}$} \\
\hline 1 & $98(63.6)$ & $82(56.6)$ & 0.24 \\
\hline 2 & $81(52.6)$ & $64(44.1)$ & 0.16 \\
\hline 3 & $73(47.4)$ & $62(42.8)$ & 0.49 \\
\hline 4 & $70(45.5)$ & $59(40.7)$ & 0.42 \\
\hline 6 & $61(39.6)$ & $56(38.6)$ & 0.91 \\
\hline
\end{tabular}

Table 3b. Only for those attending their quit day (No.1\%)

\begin{tabular}{|l|l|l|l|}
\hline Weeks of abstinence & Exercise group $(\mathrm{n}=129)$ & Control group $(\mathrm{n}=128)$ \\
\hline 1 & $98(76.6)$ & $82(63.6)$ & 0.03 \\
\hline 2 & $81(63.3)$ & $64(49.6)$ & 0.03 \\
\hline 3 & $73(57.0)$ & $62(48.1)$ & 0.17 \\
\hline 4 & $70(54.7)$ & $59(45.7)$ & 0.17 \\
\hline 6 & $61(47.7)$ & $56(43.4)$ & 0.53 \\
\hline
\end{tabular}


Table 4. Mean (SD) ratings of tobacco withdrawal symptoms (range=1-5)

(a. =baseline; b., c., $d .=$ following one, two and three weeks of smoking abstinence, respectively.)

Table 4a. For those abstinent from smoking for one week

$(\mathrm{P}<.05$ for all items for exercise/control comparison between time a. and b.)

\begin{tabular}{|c|c|c|c|}
\hline Withdrawal item & \multicolumn{2}{|c|}{ Exercise Group $\mathrm{N}=98$} & \multirow[t]{9}{*}{ Control Group $\mathrm{N}=82$} \\
\hline Tension a. & $2.5(1.1)$ & $2.5(1.2)$ & \\
\hline Tension $\mathrm{b}$. & $2.4(0.9)$ & $2.8(1.2)$ & \\
\hline Anxiety a. & $2.4(1.1)$ & $2.2(1.2)$ & \\
\hline Anxiety b. & $2.1(0.8)$ & $2.4(1.3)$ & \\
\hline Stress a. & $2.5(1.3)$ & $2.4(1.3)$ & \\
\hline Stress b. & $2.3(1.0)$ & $2.6(1.3)$ & \\
\hline Happiness a. & $3.1(0.9)$ & $3.3(1.1)$ & \\
\hline Happiness b. & $3.2(0.9)$ & $3.1(0.8)$ & \\
\hline
\end{tabular}


Table 4b. For those abstinent from smoking for two weeks (irritability, N=145) and three weeks (restlessness, $N=135)(\mathrm{P}<.05$ for for exercise/control comparison between time a. and b./c. (irritability), and between time a. and b./c./d. (restlessness) )

\begin{tabular}{|l|l|l|}
\hline Withdrawal item & \multicolumn{2}{|l|}{ Exercise Group } \\
\hline Irritability a & $2.2(1.0)$ & $1.9(0.7)$ \\
\hline Irritability b & $2.6(1.1)$ & $2.6(1.0)$ \\
\hline Irritability c & $2.3(0.9)$ & $2.5(1.1)$ \\
\hline Restlessness a & $2.6(1.2)$ & $2.1(1.0)$ \\
\hline Restlessness b & $2.7(1.1)$ & $2.6(0.9)$ \\
\hline Restlessness c & $2.4(1.0)$ & $2.4(1.0)$ \\
\hline Restlessness d & $2.2(1.1)$ & $2.2(1.0)$ \\
\hline
\end{tabular}


Figure 1. Participant Flow 